\title{
MATERNAL INFECTION WITH PARVOVIRUS B19 LEADING TO FETAL HYDROPS AND INTRAUTERINE DEATH - CASE PRESENTATION
}

\author{
Mărginean Claudiu', Pușcașiu Lucian', Molnar Varlam Claudiu', Rugină Cosmin² \\ ${ }^{1}$ University of Medicine and Pharmacy Tîrgu Mureș, Department of Obstetrics and Ginecology I \\ ${ }^{2}$ The County hospital Piatra Neamt-Department of Obstetrics and Ginecology
}

\begin{abstract}
Parvovirus B19 belongs to the Parvoviridae family, Erythrovirus type, and it presents cytotoxicity on the erythroblast human line leading to severe anemia. We present the case of a 35-year-old woman, at the 3rd pregnancy, with a first trimester abortion in her history and a physiological birth, who presented to the specialty check-up at 20 gestational weeks, associating the signs of a respiratory infection, without any other pathologies until this gestational age. The laboratory tests and the fetal ultrasound did not point out any pathological elements, therefore the patient was sent home with the recommendation to come back after 2 weeks for reevaluation, when the fetal ultrasound revealed fetal hydrops and severe anemia, and after 24 hours fetal asystole. Maternal serology pointed out recent infection with Parvovirus B19. The particularity of this case consists in the appearance of relative rare fetal infection in the second trimester of pregnancy in the case of a physiological, monitored pregnancy, with unfavorable prognosis and fulminant death towards intrauterine death.
\end{abstract}

Keywords: maternal infection, Parvovirus B19, fetal hydrops, intrauterine death

\section{INTRODUCTION}

Parvovirusul B19 is and DNA virus with one strand, being the only member of the Parvoviridae family, Erythrovirus type that can present pathogen character for the human being (1). This virus presents cytotoxicity on the progenitor cells of the erythroblast line, being a potent inhibitor of erythropoiesis (2). Maternal infection with this virus can produce severe fetal complications, such as: fetal anemia, neurological impairment, fetal hydrops, and even intrauterine death (2). The diagnosis of this infection is established on the detection of IgM and IgG anti-Parvovirus B19 antibodies in the maternal blood, but the physician must take under con- sideration that in the first 7 days after the infection, both, IgM and IgG antibodies can be negative, the patient being in the „immunological window" (2). Another important aspect that can represent a challenge for the diagnosis is that at the moment of discovering the fetal hydrops, the IgM type antibodies can already present much lower levels, or very rarely, even undetectable (2). On the other hand, the testation of the fetal serology anti-Parvovirus is not a useful diagnostic tool for establishing if the fetus is infected or not due to the fact that the fetus does not produce antibodies against the virus, thus the examinations from the fetal or neonatal serum will be reserved only for the detection of the viral DNA through Polymerase Chain-Reaction (PCR), 
the only certain method for establishing the presence of the fetal infection (2). When the suspicion of a fetal infection with Parvovirus B19 is raised, the fetal ultrasound assessment is mandatory in order to identify the signs of anemia or fetal hydrops. Therefore, the physician will assess the vascular flow in different fetal vessels, that will have a hyperdynamic pattern, but the first vessel that will respond to the fetal anemia is the medium cerebral artery (2). The increase of the systolic velocity in the medium cerebral artery presents increased sensibility in order to identify the fetal anemia caused by the infection with Parvovirus B19 (3). Fetal hydrops caused by anemia is initially manifested through ascites, the increase in volume and the thickening of the fetal miocardium, evolving towards anasarca, pleural effusion and placental edema (2). The treatment of the fetal infection with Parvovirus B19 supposes intrauterine transfusion that corrects the fetal anemia, being able to reduce significantly the mortality related to this pathology (2), with the condition to be administered as soon as possible, before the development of complications (4). In most of the cases, a single transfusion is enough for the fetal recovery, but it can take weeks until the complete remission of all the signs of fetal hydrops (2). The first mandatory step in front of a possible infection with Parvovirus during pregnancy, is to determine the maternal serological for this virus. If the mother presents only IgG positive antibodies, she will be assured that she already had the infection and therefore it won't have any consequences on the fetus. On the other hand, if the mother presents only IgM positive antibodies against Parvovirus B19, the fetus will be monitored by ultrasound weekly for approximately 12 weeks after the exposure (2). Once the signs of fetal infection have appeared, the intrauterine transfusion should be taken under consideration, or inducing the labor in the case of a close to term pregnancy (5). Regarding the prognosis, it is favorable in case of the fetuses that survived an intrauterine transfusion and recovered after a fetal hydrops, having an adequate neurological development (6).

\section{CASE PRESENTATION}

We present the case of a 35-year-old patient, at the $3^{\text {rd }}$ pregnancy, having the gestational age of 20 weeks, who presented for a specialty medical check-up associating the signs of a respiratory infection. The personal history revealed a spontaneous first trimester abortion and a normal birth.

The clinical exam performed in the moment of presentation revealed relatively good general status, a temperature of $37.7^{\circ} \mathrm{C}$, catarrh of the yeas and nose, rhinorrhea and dry cough. The local exam indicated a closed, long col, without any loss of amniotic fluid or blood, uterine fundus close to the umbilical scar, normal basal uterine tonus and absent uterine contractions, present active fetal movements. The obstetrical ultrasound exam pointed out a normal fetal morphology, normal quantity of the amniotic fluid, fundal placenta first degree of maturation, fetal heart in sinus rhythm, 136 beats/minute. Due to the above mentioned signs and symptoms, we decided to perform total CBC count and urinary exam, but they were in normal range. The history of the actual pregnancy did not reveal any pathological elements, the patient presenting periodically to the clinical and ultrasound specialty examinations, and the laboratory tests performed during the pregnancy were in normal range. In the first trimester, the serum tests for the contagious diseases pointed out only positive IgG antibodies for Rubella and herpes virus, negative for Toxoplasma and Cytomegalovirus, and the IgM type negative for all the four. After evaluating clinically and paraclinically the patient, she received the following recommendations: paracetamol in case of high fever, vitamin $\mathrm{C}$ and specialty check-up after 2 weeks. 


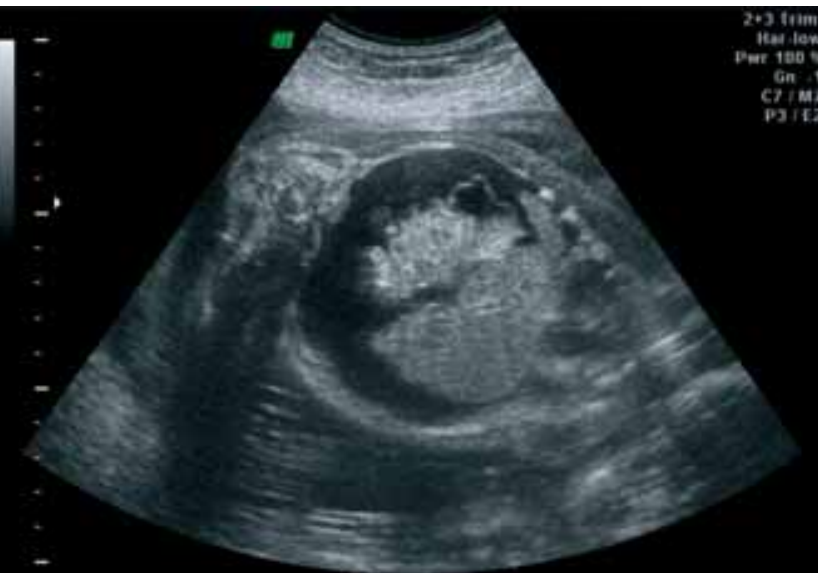

Figure 1. Fetal ascites

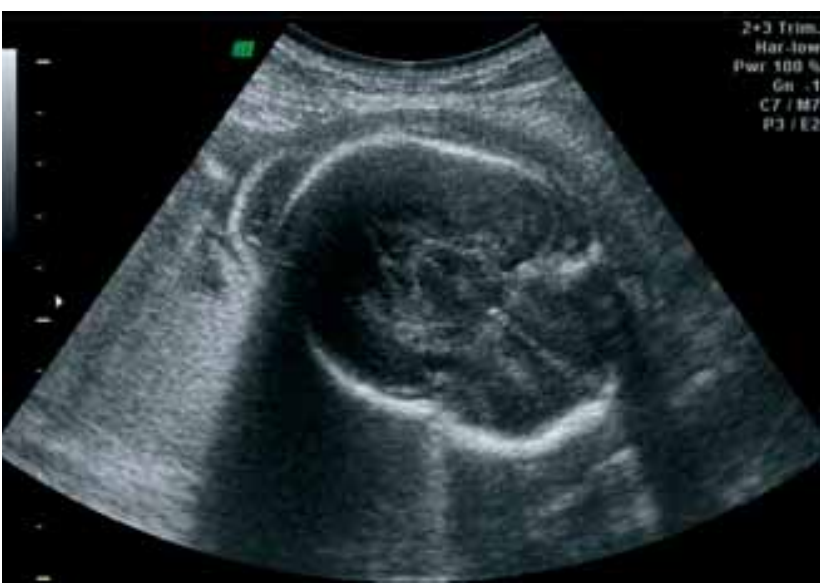

Figure 2. Edema of the fetal scalp

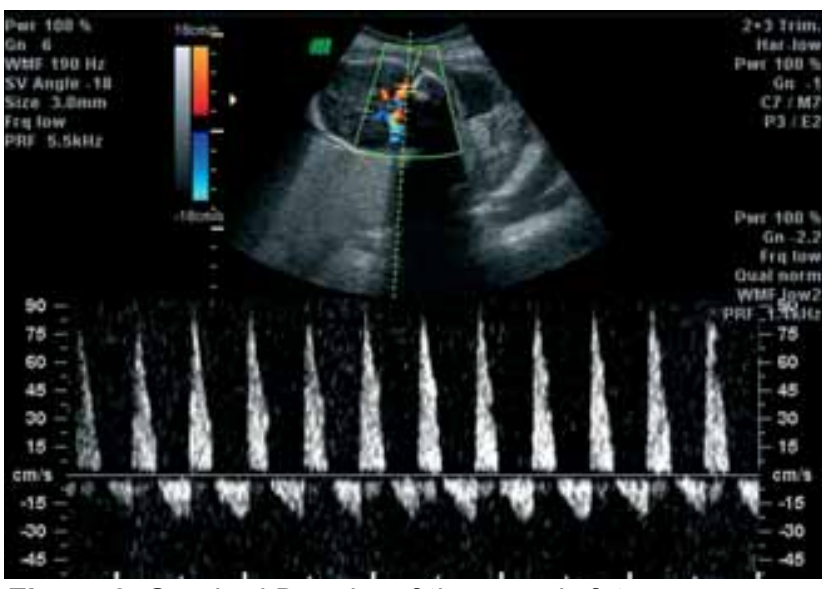

Figure 3. Cerebral Doppler of the anemic fetus

The patient came back at 23 gestational week for the obstetrical check-up for the decrease of the intensity and frequency of the active fetal movements. The clinical exam was did not reveal any pathological elements, and also the obstetrical one. The ultrasound exam revealed a

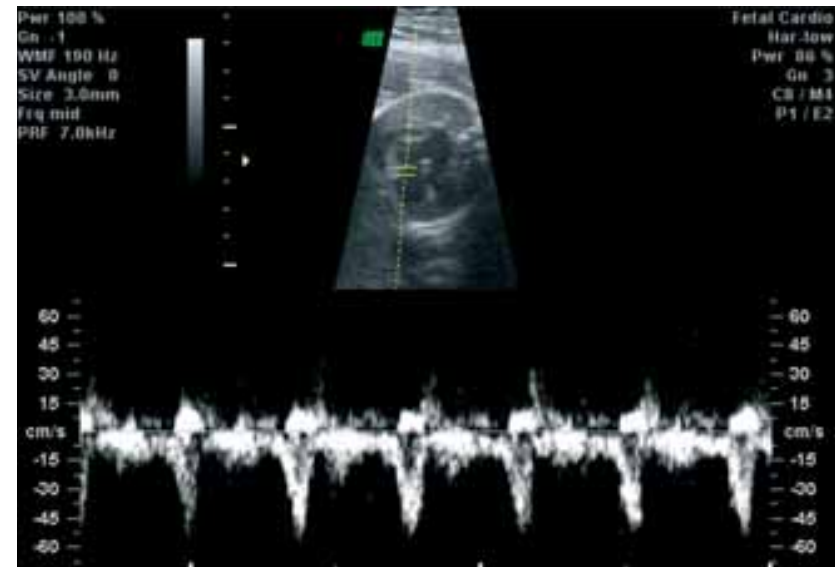

Figure 4. Regurgitation on the fetal tricuspid valve in case of hydrops

fetus in hydrops, with massive ascites (Fig. 1), edema of the scalp (Fig. 2), the signs of cerebral Doppler indicated a severe anemia (Fig. 3) and the specific volume overloading with regurgitation on the tricuspid valve (Fig. 4).

After 24 hours, we repeated the fetal ultrasound, which revealed fetal asystole. We tried to identify a maternal infection, therefore we repeated the CBC count and the urinary exam, but they remained in normal range. We also performed TORCH serology in order to identify a recent infectious disease, but the values were the same as in the first trimester. Therefore, we widened the investigations spectrum in order to exclude a less frequent infection, and we identified positive IgM antibodies anti-Parvovirus B19, pointing out a recent maternal infection with this virus. The abortion induced by drugs with oxytocin perfusion led to the expulsion after 12 hours of a conception product of approximately $1 \mathrm{~kg}$, male gender sex, with the typical edematous aspect and without signs of maceration or obvious anomalies. The pathological exam of the aborted fetus confirmed the presence of the subcutaneous generalized fluid, and in all physiological cavities, of a severe enlarged liver and spleen, and of a very increased volume placenta, all these being signs of fetal infection. The evolution of the patient after the abortion was favorable.

The particularity of this case consists in the appearance of relative rare fetal infection in the 
second trimester of pregnancy in the case of a physiological, monitored pregnancy, with unfavorable prognosis and fulminant death towards intrauterine death

\section{DISCUSSIONS}

Infection with Parvovirus B19 is common worldwide, presenting a peak of annual incidence during spring, with the appearance of epidemics approximately once at 4 years (7). The prevalence of the IgG antibodies anti-Parvovirus B19 increased at the same time with the age, thus, in children between 1 and 5 years of age, it varies between 2 and 15\%, in adults and old people reaches over $85 \%$ (8). Nevertheless, in the case of our patient, the IgG antibodies antiParvovirus B19 were negative despite her age of 35 years. In case of maternal infection with Parvovirus B19, the vertical transmission appears in a percentage of $33-51 \%$ of the cases, and the risk of unfavorable fetal effects is approximately $10 \%(9,10)$. Therefore, a study performed by Bosman underlines the fact that the infection with Parvovirus B19 causes 48 fetal deaths every year in Netherlands (7). The case presented above also reveals a fetal death due to the infection with Parvovirus B19. This infection can appear in every moment during the pregnancy. Therefore, the most frequent cases of intrauterine death caused by this infection were reported between 20-24 gestational weeks, but there were also described cases of fetal death at 10 gestational weeks, and also 41 gestational weeks (10). In the case of our patient, the fetal death occurred at the 23 gestational weeks, similarly to the data from the literature. The risk of the fetal hydrops development is of 3.9\% during the pregnancy, but it can reach $7.1 \%$ when the infection occurs between the weeks 13 and 20 of gestation (2), similarly in our case. Enders et al underlined the fact that the peak of the fetal hydrops incidence associated to the infection with Parvovirus B19 appears between 17-24 gestational weeks (4). Parvovirus B19 presents also cardiac tropism being able to cause myocarditis and to aggravate the fetal cardiac failure (11). Nevertheless, the fetal infection does not always lead to fetal complications (12). Intrauterine transfusion with erythrocytes is an effective method in case of fetal anemia induced by the infection with Parvovirus and it can increase the fetal survival rate with up to $83.5 \%$ (13), still half of the fetuses that receive intrauterine transfusion also develop severe thrombocytopenia whose mechanism is unclear (14). Therefore, platelets transfusion should also be taken under consideration in case of fetuses with thrombocytopenia. On the other hand, the platelets transfusion will lead to the significant increase of the administered volume to a fetus already with fetal hydrops, cardiac impairment and severe anemia due to Parvovirus, therefore the benefits of this therapeutic measure should be weighed against to the risk that are implied (15). In the above presented patient, the intrauterine transfusion was not administered due to the severe fetal impairment and also due to the identification of fetal asystole. The vaccination against Parvovirus B19 with a vaccine described by Ballou et al, can represent a very effective method for preventing the fetal infection in case of pregnant women with negative IgG antibodies (16).

\section{CONCLUSIONS}

The infection with Parvovirus B19, though frequent among the world wide population, represents a major problem during pregnancy due to the fact that it can lead to severe fetal complications such as fetal hydrops, severe anemia, cardiac failure, and even intrauterine death. 


\section{REFERENCES}

1. Lukashov V.V., Goudsmit J. Evolutionary relationships among parvoviruses: virus-host coevolution among autonomous primate parvoviruses and links between adeno-associated and avian parvoviruses. J Virol, 2001 Mar, 75 (6):2729-40.

2. de Jong E.P., de Haan T.R., Kroes A.C.M., Beersma M.F.C., Oepkes D., Walther F.J. Parvovirus B19 infection in pregnancy. J Clin Virol Off Publ Pan Am Soc Clin Virol, 2006 May, 36 (1):1-7.

3. Cosmi E., Mari G., Delle Chiaie L., Detti L., Akiyama M., Murphy J., et al. Noninvasive diagnosis by Doppler ultrasonography of fetal anemia resulting from parvovirus infection. Am J Obstet Gynecol, 2002 Nov, 187 (5):1290-3.

4. Enders M., Weidner A., Zoellner I., Searle K., Enders G. Fetal morbidity and mortality after acute human parvovirus B19 infection in pregnancy: prospective evaluation of 1018 cases. Prenat Diagn, 2004 Jul, 24 (7):513-8.

5. Cohen B.J., Kumar S. Parvovirus B19 infection in pregnancy. Fetal Matern Med Rev, 2005 May, 16 (2):123-150.

6. Dembinski J., Haverkamp F., Maara H., Hansmann M., Eis-Hübinger A.M., Bartmann P. Neurodevelopmental outcome after intrauterine red cell transfusion for parvovirus B19-induced fetal hydrops. BJOG Int J Obstet Gynaecol, 2002 Nov, 109 (11):1232-4.

7. Bosman A., Wallinga J., Kroes A.C.M. Fifth disease every four years: parvovirus B19. Infect Bull, 2002, 6:215-9. accessible at: http://www.rivm.nl/infectieziektenbulletin/bul1306/parvo.html.

8. Heegaard E.D., Brown K.E. Human parvovirus B19. Clin Microbiol Rev, 2002 Jul, 15 (3):485-505.

9. Chisaka H., Morita E., Yaegashi N., Sugamura K. Parvovirus B19 and the pathogenesis of anaemia. Rev Med Virol, 2003 Dec, 13 (6):347-59.
10. Norbeck O., Papadogiannakis N., Petersson K., Hirbod T., Broliden K., Tolfvenstam T. Revised clinical presentation of parvovirus B19-associated intrauterine fetal death. Clin Infect Dis Off Publ Infect Dis Soc Am, 2002 Nov 1, 35 (9):1032-8.

11. Morel O., Chagnaud S., Laperrelle J., Clément D., Malartic C., Akerman G., et al. Parvovirus B19 et grossesse: revue de la littérature. Gynécologie Obstétrique Fertil, 2007 Nov, 35 (11):1095104.

12. Brochot C., Debever P., Subtil D., Puech F. Quelles surveillance et thérapeutique appliquer en cas de contage avec le Parvovirus B19 en cours de grossesse? Gynécologie Obstétrique Fertil, 2008 Feb, 36 (2):204-11.

13. Rodis J.F., Borgida A.F., Wilson M., Egan J.F., Leo M.V., Odibo A.O., et al. Management of parvovirus infection in pregnancy and outcomes of hydrops: a survey of members of the Society of Perinatal Obstetricians. Am J Obstet Gynecol, 1998 Oct, 179 (4):985-8.

14. de Haan T.R., van den Akker E.S.A., Porcelijn L., Oepkes D., Kroes A.C.M., Walther F.J. Thrombocytopenia in hydropic fetuses with parvovirus B19 infection: incidence, treatment and correlation with fetal B19 viral load. BJOG Int J Obstet Gynaecol, 2008 Jan, 115 (1):76-81.

15. Argoti P.S., Bebbington M., Adler M., Johnson A., Moise K.J. Serial intrauterine transfusions for a hydropic fetus with severe anemia and thrombocytopenia caused by parvovirus: lessons learned. AJP Rep, 2013 Oct, 3 (2):75-8.

16. Ballou W.R., Reed J.L., Noble W., Young N.S., Koenig S. Safety and immunogenicity of a recombinant parvovirus $B 19$ vaccine formulated with MF59C.1. J Infect Dis, 2003 Feb 15, 187 (4):675-8. 